\title{
Parental Autonomy Support and Social Competence in Chinese Emerging Adults: the Mediation Role of Social Desirablity
}

\author{
Chunhua $\mathrm{Ma}^{1, \mathrm{a}}$, Youpeng Wang ${ }^{2}$ \\ ${ }^{1}$ Department of Psychology, Northwest Minzu University, China \\ ${ }^{2}$ Lanzhou Petrochemical College of Vocational Technology, China
}

\begin{abstract}
Aims: The current study aimed to examine the relationship between parental autonomy support and social competence among Chinese emerging adults, and explore whether social desirablity plays a mediating role between parental support and social competence. Methods: This study used cross-sectional and correlational design. Participants were 386 Chinese college students ( $72.8 \%$ girls) aged between 18 and 25 years. Data was collected via self-report questionnaires, including parental autonomy support (Genevie've A. Mageau, 2015), social desirablity (Karl Schuessler et al., 1978) and social competence(Valkenburg \& Peter, 2008). Results: Structural equation modeling analysis controlling for age, gender and SES showed that (a) There was a significant positive correlation between parental autonomy support, social desirablity, and social competence; (b) Parental autonomy support was positively predicted to social desirablity and social competence; Social desirablity was positively predicted to social competence; (c) Social desirablity mediated the relationship between parental autonomy support and social competence.
\end{abstract}

\section{Introduction}

Autonomy Support means that individuals feel the support from important others (such as teachers and parents) for their free choice and independent decision, it help people get valuable information, gain recognition of emotional experience and feel smaller pressure (Deci \& Ryan, 1987). Parental support is an important environmental factor that effect the development of adolescents, many studies have shown that adolescents with parental support have higher motivation (Gillet, Vallerand \& Lafrenière, 2012), better academic performance (Grolnick, 2009), better levels of life satisfaction and self-esteem (Chirkov \& Ryan, 2001). Independent support effect the social development of college students, because university stage is an important period for students' social development, their will face many pressures from study and employment. Social competence is an aspect of college students' sociality. Parental support can effectively predict college students' social competence.

Social desirablity refers to a motivational tendency of individuals to adapt their attitudes and behaviors to social requirements in self-evaluation, the purpose is to gain recognition and maintain self-esteem that is suitable for social needs. Which premise is people understand the values, requirements, and norms of their culture and society. The way to obtain social desirablity is to make themselves conform to the majority of people in attitudes and behaviors. Social desirablity as a social factor a Corresponding author: mch@xbmu.edu.cn affecting the development of college students' attitudes and behaviors, it will be effect on the development of students' social abilities.

Social competence is a concept in psychology, it is an ability to understand people's feelings, thoughts and behaviors in an interpersonal situation, and make appropriate behaviors on this state. Social competence includes a range of knowledge, experience, and abilitys to explain social information and solve interpersonal problems. Schneider (1996) define social competence as the ability to act effectively in social interactions, which is the basic ability to motivate individuals to achieve their social goals. Baron and Markman (2003) define social competence in entrepreneurial research as the effectiveness of interaction between actors and others, and the integration of multiple social skills.

In the past studies, the relationship between parental support and social development of students was verified. However, the relationship between parental support, social desirablity and social competence was rarely discussed. In this study, we use college students conduct surveys explored the relationship between parental support, social desirablity, and social competence, and further explore whether social desirablity plays a mediating role between parental support and social competence.

The present study explored the mechanisms underlying the association between parental autonomy support and social competence in emerging adults. 
Specifically, we examined a mediation model to test two hypotheses:

Hypothesis 1. There are significant correlations between parental autonomy support, social desirablity and social competence: parental autonomy support would be positively related to social desirablity, which in turn would be related to social competence.

Hypothesis 2. Social desirablity would mediate the relationship between parental autonomy support and social competence in emerging adults. The association between parental autonomy support and social competence would be stronger for individuals with high social desirablity than for those with low social desirablity.

\section{Methods}

\subsection{Participants}

Participants in this study involved 386 Chinese emerging adults aged 18 to 25 years $(\mathrm{M}=21.28, \mathrm{SD}=.45 ; 72.8 \%$ girls, $27.2 \%$ boys), who were college students attending 1 th to 4 th (sophomore and junior consist of $53.1 \%$ sample size, as freshman and senior face transition and high pressure) in Lanzhou, China.

\subsection{Measures}

\subsubsection{Parental autonomy support}

Parental autonomy support was measured by parental autonomy support scale (Genevie ve A. Mageau2015), which was a 24-item scale. Participants were asked to rate each item from 1 (Definitely does not apply to me) to 5 (Definitely applies to me) on the Likert scale. The average score of 24 items was calculated, with a higher score indicating a higher level of parental autonomy support. Cronbach's $\alpha$ was. 85 for perseverance and. 80 for consistency in the present study.

\subsubsection{Social competence}

Social competence was measured using the Social competence scale (Valkenburg, Patti M., \& Peter, Jochen. (2008). The scale is a self-report inventory measuring social competence among college students. It has four dimensions: Initiation, Supportiveness, Self-disclosure and Assertiveness. It contains 19 items (e.g., "Start a conversation with someone you did not know very well?") that use a 5-point scale ranging from 1 (Very difficult) to 5 (Very easy). The scale was scored such that high scores identified students who were have high level of social competence. Cronbach's $\alpha$ was .85 for perseverance and .87 for consistency in the present study.

\subsubsection{Social desirablity}

Social desirablity measure (Karl Schuessler,1978) used in this study, It contains 16 items, that use a 5-point scale ranging from 1 (very much unlike me) to 5 (very much likeme'). Participants were asked to rate each item on the Likert scale. Cronbach's $\alpha$ was .75 for perseverance and .80 for consistency in the present study.

\subsubsection{Controlling variables}

Age and gender. Participants were asked to complete a questionnaire providing information on their year of birth and gender. As the method of calculating age is inconsistent from the various region in China, we received the age using the date of data collection minus year of birth uniformly.

SES was assessed via maternal and paternal education background and occupation. In regard with education, it provides four choices for children: (1) middle school graduation or lower, (2) high school graduation, (3) bachelor degree graduation, and (4) master degree graduation or higher. However, as for parental occupation, it provides seven choices base on occupational classification criteria in China (adapted from Shi \& Shen, 2007).

\subsection{Statistical analysis}

Based on SPSS 21.0 (IBM Corporation, Armonk NY, USA), We first conducted the analyses of descriptive statistics and correlation. Then, we examined the moderation model using the SPSS macro PROCESS proposed by Hayes (2013).

\section{Results}

\subsection{Preliminary analyses}

The results of the correlation analysis are presented in Table1 that there is significant correlation between parental autonomy support, social desirablity and social competence. parental autonomy support would be positively related to social desirablity, which in turn would be related to social competence. Hypothesis 1 was supported.

Table1. Descriptive statistics and intercorrelations between variables

\begin{tabular}{ccccccc}
\hline Variables & M & SD & Range & 1 & 2 & 3 \\
\hline Parental Autonomy Support & 102.920 & 15.349 & $1-5$ & - & & \\
Social Competence & 64.254 & 9.524 & $1-5$ & $.251^{* *}$ & - & - \\
Social Desirablity & 78.552 & 12.124 & $1-5$ & $.376^{* *}$ & $.487^{* *}$ & - \\
\hline
\end{tabular}

Note. $* * \mathrm{p}<.01$ 


\subsection{Testing for the mediation model}

As can be seen from Table 2, after controlling for gender and age, parental autonomy support positively predicted social desirablity $(\beta=0.30, p<0.001)$ and parental autonomy support positively predicted social competence $(\beta=0.16, p<0.001)$, social desirablity positively predicted social competence $(\beta=0.39, p<0.001)$. It mean social desirablity mediate the relationship between parental autonomy support and social competence. Hypothesis 2 was supported.

Table 2. A Moderated Mediation analysis.

\begin{tabular}{llllll}
\hline Outcome variables & $\begin{array}{l}\text { Independent } \\
\text { variables }\end{array}$ & $\beta$ & SE & $\mathrm{t}$ & $\mathrm{p}$ \\
& Age & 0.15 & 0.18 & 0.85 & 0.40 \\
Social Desirablity & Gender & 1.98 & 1.31 & 1.51 & 0.13 \\
& PAS & 0.30 & 0.04 & 7.95 & 0.00 \\
Social Competence & Age & -0.13 & 0.14 & -0.90 & 0.37 \\
& Gender & -0.27 & 1.08 & -0.25 & 0.81 \\
& PAS & 0.16 & 0.03 & 5.01 & 0.00 \\
& SD & 0.39 & 0.04 & 10.95 & 0.00 \\
\hline
\end{tabular}

\section{Discussion}

This study found that there is a significant positive correlation between parental support, social desirablity, and social competence. It mean that when emerging adults get more support from their parents, it is it is easier to make their attitudes and behaviors consistent with social requirements, and they often have better social competencies.

The regression analysis found that parental autonomy support positively predicted Chinese emerging adults' social desirablity and social competence. It mean that when the emerging adults have more support, they may have more interpersonal skills in social activities, and they can handle various problems in the their day life. parental support has a positive impact on Chinese emerging adults' social desirablity and social competence. Emerging adults' attitudes and behaviors required by the society are influenced by the parents, and the students are encouraged and supported by the parents. Therefore, in cultivating the attitudes and behaviors of college students' social desirablity, we should know the students' family situation, especially the attitudes and emotions of the students in the process of parenting, and then for targeted education.

Through mediation analysis, social desirablity plays a full mediating role between parental autonomy and social competence. For Chinese emerging adults, the influence of parental support on social competence is achieved through social desirablity. Only when the support of parents becomes the college students' attitude and behavior, this attitude and behavior can promote the students' social desirablity, so that they can develop their talents in social activities and adapt to the social environment.Parental autonomy support was an important factor it would impact students' social competence .For Chinese emerging adults if they have high level parental autonomy support they may have high level social competence, social desirablity as a mediator varable it link parental autonomy support and social competence.

\section{Conclusion}

The aim of this paper is to explore the mechanisms underlying the association between parental autonomy support and social competence in emerging adults. Specifically, we examined social desirablity as a mediation variable. In summary, this paper can draw the following conclusions: (1) There was a significant positive correlation between parental autonomy support, social desirablity, and social competence; (2) Parental autonomy support was positively predicted to social desirablity and social competence; Social desirablity was positively predicted to social competence; (3) Social desirablity mediated the relationship between parental autonomy support and social competence.

\section{Acknowledges}

The current study was supported by "the Fundamental Research Funds for Central Universities" [Project No. zyz2012038] and "the Fundamental Research Funds for the Central Universities" in 2017 (Project No: 31920170096), we want to thank all the participants and Hui-xue Lao for helping collect data in China.

\section{References}

1. Baron R. A, Markman G. D. etal. Beyond social capital: the role of entrepreneurs. social competence in their financial success. Journal of Business Venturing [J] 2003, 18(1), 41-60

2. Geneviève A. Mageau, Validation of the Perceived Parental Autonomy Support Scale (P-PASS) Canadian Journal of Behavioural Science / Revue canadienne des sciences du comportement [J], 2015, Vol. 47, No. 3, 251-262

3. Ma D H, Li Y, College Students' Social Development Guidance. Modern Education Science [J], 1998, 15(1), 100-103 
4. Measuring Responding Desirably with Attitude-Opinion Items. Social Psychology [J]. Vol. 41, No. 3 (Sep., 1978), 224-235

5. Ryan R. M, Deci E L, Grolnick W. S, La Guardia J. G, et al. The significance of autonomy and autonomy support in psychological development and psychopathology [J]. In D.Cicchetti \& D.J.Cohen (Eds.), Developmental psychopathology, Hoboken, NJ,US, 2006: 795-849

6. Schneider R J, Ackerman P L, Kanfer R. et al. To act wisely in human relations: exploring the dimensions of social competence. Personality and Individual Differences [J]. 1996, 21(4), 469-481

7. Tang Q, Fang X. L, et al. The relationship between parents and teachers' independent support and the development of high school students, psychological development and education [J], 2013, (6), 604-615, Valkenburg, P. M., \& Peter, J. et al. Social Competence Scale Version Attached: Full Test. Communication Research [J], 2008 Vol 35(2), 208-231.

8. Xu W, Controversy, Application and Prospect of Social desirablity, Nankai Journal Philosophy and Social Sciences Edition) [J], 2014,3,152-160

9. Yan S L, Narcissism and Self-Esteem: The Mediating Role of Social desirablity. Applied Psychology [J], 2009, 15(1), 056-061 